\section{P46 (continued)}

$(\mathrm{p}<0.0001)$, and soda $(\mathrm{p}<0.0001)$ than Philadelphia YRBS respondents. Post-intervention, these trends continued and reductions were seen in the number of students reporting no fruit $(\mathrm{p}=0.0373)$ or vegetable $(\mathrm{p}=0.0117)$ consumption. Additionally, an increase was seen in students reporting no soda consumption $(\mathrm{p}=0.0014)$.

Conclusions and Implications: Our intervention positively impacted the eating behaviors of high school students receiving this curriculum. The evidence supports this curriculum as an effective tool in helping to improve healthy eating behaviors in Philadelphia's youth and could be an effective tool for high school students throughout the nation and within SNAP-Ed.

Funding: Supplemental Nutrition Assistance Program Education

\section{P47 Child Health Assessment in the Pacific (CHAP) Summer Fellowship Program}

Marie Kainoa Fialkowski,PhD, RD, LDN, mariekf@hawaii. edu, University of Hawaii at Manoa, 2500 Campus Road, Honolulu, HI 96822; M. Sandlin, PhD;

M. Hattori-Uchima, PhD, RN, University of Guam;

M. Weichhaus, PhD, Chaminade University; P. Coleman, Northern Marianas College; R. Novotny, PhD, RDN, LDN, University of Hawaii at Manoa

Objective: Child Health Assessment in the Pacific (CHAP) Summer Fellowship program has 3 objectives: build Pacific regional capacity in early childhood nutrition and health assessment; create a network of individuals working to monitor and prevent early childhood health disparities; evaluate opportunities to sustain training in early childhood nutrition and health assessment.

Target Audience: Thirty undergraduate students from land-grant colleges and other degree seeking institutions in Hawai' $i$, the Commonwealth of the Northern Mariana Islands, Chuuk, and Guam that are enrolled in programs such as nutrition, nursing, early childhood education, and public health and committed to working in the region. Theory, Prior Research, Rationale: Few professionals in the region have the training or resources to develop, deliver, monitor and evaluate childhood health assessment programs.

Description: CHAP consists of a 6-week didactic phase followed by a 6-week application phase. In the didactic phase, students complete an introductory nutrition course and a health assessment course adapted for the Pacific online followed by a one-week hands-on training. In the application phase, students conduct a place-based miniproject. Each student will be matched with a mentor.

Evaluation: CHAP will be evaluated based on the context, input, process, and product (CIPP) model for evaluation. Quantitative data focuses on content competencies and implementation processes while qualitative data focuses on implementation process, participant and stakeholder perceptions, and overall value.

Conclusions and Implications: CHAP has the potential to contribute to a regional effort to train an interdisci- plinary team of professionals in diverse fields that will support childhood health assessment and monitoring in the Pacific.

Funding: USDA

\section{P48 B.A.L.A.N.C.E.: Evaluating the Effectiveness of a 14-Week Worksite Wellness Program on Reducing Metabolic Syndrome Risk Factors}

Melissa Manni, MS,mmanni@okstate.edu, Oklahoma State University, 921 E Echo Mountain Drive, Stillwater, OK 74075; E. Lohrman, MS, RDN; G. Gates, PhD

Objective: To determine how successful the 14-week B.A.L.A.N.C.E. program was in reducing risk factors of metabolic syndrome, and determine if participants viewed this program as helping build a healthy lifestyle.

Design, Setting, Participants, and Intervention: Eighty-nine university employees at risk for metabolic syndrome participated in the program between 2011-2014. Participants received three individual counseling sessions with an $\mathrm{RD}$, attended $\geq 10$ nutrition education sessions, and had access to a personal trainer for $\geq 60$ minutes/ week. After the 14-week intervention, participants attended $\geq 5$ monthly group meetings until the one-year mark. Participants were invited to complete a perceptions survey after completion of the program; 40 completed the survey.

Outcome Measures and Analysis: BMI, abdominal girth, cholesterol, triglycerides, HDL and LDL cholesterol, blood pressure (BP), and blood glucose were measured at week one, week 14 and one year follow-up. Fitness was assessed at baseline and week 14 using paired t-tests.

Results: Paired t-tests showed significant reductions in BMI, girth, triglycerides, blood glucose, systolic and diastolic BP, flexibility, muscular strength, and VO2 Max after 14 weeks. From baseline to one-year, paired t-tests found significant reductions in BMI, girth, blood glucose, and systolic BP. Most participants agreed or strongly agreed that participation was beneficial, they would recommend this program to other employees, and they understood more about nutrition and exercise.

Conclusions and Implications: Results show a shortterm worksite wellness program was effective in reducing metabolic syndrome risk factors, and participants perceived the program as being beneficial to their health. Funding: None

P49 Who is Aware of and Has Tried to Use MyPyramid? An Examination of Variables From the Most Recent NHANES Consumer Behavior Phone Follow-Up Module-Child (FCBS).

Erin Green, RD, emg232@cornell.edu, Cornell University, 417 Savage Hall-Division of Nutritional Sciences, Cornell University, Ithaca, NY 14850; A. Gaines, PhD;

J. Dollahite, PhD 